BNL- 61920

AD $/$ RHIC-133

Informal Report

\title{
Hybrid Helical Snakes and Rotators for RHIC
}

\author{
E. D. Courant
}

June 1995

DISCLAIMER

\begin{abstract}
This report was prepared as an account of work sponsored by an agency of the United States Government. Neither the United States Government nor any agency thereof, nor any of their employees, makes any warranty, express or implied, or assumes any legal liability or responsibility for the accuracy, completeness, or usefulness of any information, apparatus, product, or process disclosed, or represents that its use would not infringe privately owned rights. Reference herein to any specific commercial product, process, or service by trade name, trademark, manufacturer, or otherwise does not necessarily constitute or imply its endorsement, recommendation, or favoring by the United States Government or any agency thereof. The views and opinions of authors expressed herein do not necessarily state or reflect those of the United States Government or any agency thereof.
\end{abstract}

\section{R H I C P R O J C T}

\author{
Brookhaven National Laboratory \\ Associated Universities, Inc. \\ Upton, NY 11973
}

Under Contract No. DE-AC02-76CH00016 with the
UNITED STATES DEPARTMENT OF ENERGY

MASTER 


\section{DISCLAIMER}

Portions of this document may be illegible in electronic image products. Images are produced from the best available original document. 


\title{
HYBRID HELICAL SNAKES AND ROTATORS FOR RHIC
}

\author{
E. D. Courant
}

June 13, 1995

The spin rotators and Siberian snakes presently envisaged for RHIC utilize helical dipole magnets. The snakes and the rotators each consist of four helices, each with a full twist $\left(360^{\circ}\right)$ of the field. Here we investigate an alternate layout, namely combinations of helical and pure bending magnet, and show that this may have advantages.

\section{Requirements.}

Each of the two RHIC rings needs two snakes, which should each rotate the spin by $180^{\circ}$ about an axis that is in the horizontal plane at $45^{\circ}$ from the longitudinal, with the two snakes located in the $Q 8-Q 9$ straight sections exactly $180^{\circ}$ apart. Because of the length of these straight section each snake should be not more than 11 meters long.

For experiments with pure helicity states we need longitudinal polarization at the beam crossing points. Therefore we must rotate the vertical spin in the arcs into a longitudinal spin at the crossing point, and back again. To this end one needs $90^{\circ}$ rotators in the long straight section between $Q 3$ and $Q 4$ on either side of the STAR and PHENIX crossing points in each ring, i.e. four rotators per ring. Since the DO and DX magnets lie between the rotator and the crossing point, and will precess the spin by an angle of $G \gamma \varphi(\varphi=3.6745$ milliradians = bending angle of DO plus DX; $G=1.7928=$ proton anomalous moment), the rotators must rotate the spin from vertical to an angle $G \gamma \varphi$ from longitudinal in the horizontal plane, which varies from $10.9^{\circ}$ at injection $(\gamma=27)$ to $101.2^{\circ}$ at $250 \mathrm{GeV}$.

The snakes and rotators should each produce zero net orbit displacement and deflection, and the maximum orbit excursion within each snake or rotator (which will be largest at injection energy) should be as small as possible.

Snakes and rotators accomplishing this can be constructed using combinations of interleaved horizontal, vertical, and/or tilted deflecting magnets, as well as helical deflecting magnets. We now review the methods for calculating the effects of these components.

\section{Conventions}

We use a coordinate system with the variables $s, x, y$ (longitudinal, transverse horizontal, transverse vertical), also designated as $x_{1}, x_{2}, x_{3}$. We use the paraxial approximation, i.e. we assume the motion is primarily along the $s$ direction, and all quantities of higher than first degree in the deviations $x$ and $y$ 
from the central reference orbit will be neglected. This enables us to use linear spin rotation matrices, which can be calculated analytically.

We consider two types of magnets: dipoles and helices.

A dipole has a constant field $B$ which may be oriented in the vertical or horizontal direction or tilted in an intermediate direction by an angle $\alpha$ from the vertical; we do not consider solenoidal (longitudinal) fields.

A helix is assumed to have fields on the axis

$$
\begin{aligned}
& B_{x}=B \cos k s \\
& B_{y}=B \sin k s
\end{aligned}
$$

where $k=2 \pi / \lambda, \lambda$ being the length of a full twist of the helix. For a right-handed helix $k$ is positive; for a left-handed helix it is negative.

Maxwell's equations require that the fields away from the axis deviate slightly from $(1)^{1}$ :

$$
\begin{gathered}
B_{x}=B_{0}\left\{\left[1+\frac{1}{8} k^{2}\left(3 x^{2}+y^{2}\right)\right] \cos k s+\frac{1}{4} k^{2} x y \sin k s\right\} \\
B_{y}=B_{0}\left\{\left[1+\frac{1}{8} k^{2}\left(x^{2}+3 y^{2}\right)\right] \sin k s+\frac{1}{4} k^{2} x y \cos k s\right\} \\
B_{s}=-k B_{0}(x \sin k s-y \cos k s)
\end{gathered}
$$

In keeping with our paraxial approximation we ignore the nonlinear parts of

(2), so that the transverse fields are still taken to be (1); however the solenoidal field of (2) may be considered.

We neglect all fringe fields, i.e. we calculate as if all fields stepped abruptly from zero to $B$ at the edges.

\section{Orbital Motion}

The Lorentz force equations, in the paraxial approximation, are

$$
x "=\frac{1}{B \rho}\left(y^{\prime} B_{s}-B_{y}\right)
$$

\footnotetext{
${ }^{1}$ J. P. Blewett and R. Chasman, J. App. Phys. 48, 2692(1977)
} 


$$
y^{\prime \prime}=\frac{1}{B \rho}\left(B_{x}-x^{\prime} B_{s}\right)
$$

Dipole, $B_{x}=B \cos \alpha, B_{y}=B \sin \alpha$ :

$$
\begin{aligned}
& x=x_{0}+\left(x_{0}^{\prime}-\frac{s-s_{0}}{2 \rho} \sin \alpha\right)\left(s-s_{0}\right) \\
& y=y_{0}+\left(y_{0}^{\prime}+\frac{s-s_{0}}{2 \rho} \cos \alpha\right)\left(s-s_{0}\right)
\end{aligned}
$$

where $\rho \equiv(B \rho) / B, B \rho$ being the magnetic rigidity of the particle.

Helix, fields as in (2):

$$
\begin{aligned}
& x=x_{0}+\left(x_{0}^{\prime}-k r_{0} \cos k s_{0}\right)\left(s-s_{0}\right)+r_{0}\left(\sin k s-\sin k s_{0}\right) \\
& y=y_{0}+\left(y_{0}^{\prime}-k r_{0} \sin k s_{0}\right)\left(s-s_{0}\right)-r_{0}\left(\cos k s-\cos k s_{0}\right)
\end{aligned}
$$

with

$$
r_{0}=1 /\left(k^{2} \rho\right)
$$

being the radius of a helical orbit in the ideal helical field (1). Note that the solenoidal component of the field, being of higher order, does not enter into the orbit in our approximation.

\section{Spin Motion}

The spin vector $\vec{S}$ precesses in a magnetic field, satisfying

$$
\frac{d \vec{S}}{d s}=\vec{S} \times \vec{\Omega}
$$

where the precession frequency vector $\vec{\Omega}$ is (BMT equation)

$$
\vec{\Omega}=\left[(1+G \gamma) \overrightarrow{B_{\perp}}+(1+G) \overrightarrow{B_{\|}}\right] / B \rho,
$$

$\overrightarrow{B_{\perp}}$ and $\overrightarrow{B_{\|}}$being the parts of the field perpendicular and parallel to the particle velocity. The spin motion is conveniently described by the SU2 spinor 
formalism ${ }^{2}$ : We use a two-component spinor $\psi$; the spin vector $\vec{S}$ is derived from the spinor by

$$
\vec{S}=\psi^{\dagger} \vec{\sigma} \psi
$$

where $\sigma_{1}, \sigma_{2}, \sigma_{3}$ are the Pauli spin matrices. The precession equation (8) is then equivalent to the spinor equation

$$
\frac{d \psi}{d s}=\frac{i}{2}(\vec{\sigma} \cdot \vec{\Omega}) \psi
$$

which has to be solved for the given field.

Dipole, $B_{x}=B \cos \alpha, B_{y}=B \sin \alpha$ :

Equation (10) is easily solved, since the coefficients are constant. The solution can be written in matrix form

$$
\psi_{2}=\mathbf{M} \psi_{1}
$$

where the SU2 matrix $M$ is

$$
\mathrm{M}=\exp \left[\frac{i}{2}\left(\sigma_{2} \cos \alpha+\sigma_{1} \sin \alpha\right)\left(s_{2}-s_{1}\right) / \rho\right]
$$

Helix:

If we take the ideal fields (1) and ignore the solenoidal field (2c) equation (10) becomes

$$
\frac{d \psi}{d s}=\frac{i}{2} \kappa\left(\sigma_{2} \cos k s+\sigma_{3} \sin k s\right) \psi
$$

where

$$
\kappa=\frac{1+G \gamma}{\rho}
$$

We note that

where

$$
\sigma_{2} \cos k s+\sigma_{3} \sin k s=\sigma_{2} e^{i k s \sigma_{1}}
$$

and transform to

\footnotetext{
${ }^{2}$ B. W. Montague, Physics Reports 113, 1 - 96(1984)
} 


$$
\varphi=\exp \left(\frac{i}{2} k s \sigma_{1}\right) \psi
$$

Then (12) becomes

$$
\frac{d \varphi}{d s}=\frac{i}{2}\left(\kappa \sigma_{2}+k \sigma_{1}\right) \varphi
$$

which is an equation with constant coefficients, and is therefore easily solved:

$$
\varphi_{2}=\exp \left[\frac{i}{2}\left(\kappa \sigma_{2}+k \sigma_{1}\right)\left(s_{2}-s_{1}\right)\right] \varphi_{1}
$$

Transforming back to $\psi$, we find the matrix solution (11) with

$$
\mathrm{M}=\exp \left[-\frac{i}{2} k s_{2} \sigma_{1}\right] \exp \left[\frac{i}{2}\left(\kappa \sigma_{2}+k \sigma_{1}\right)\left(s_{2}-s_{1}\right)\right] \exp \left[\frac{i}{2} k s_{1} \sigma_{1}\right]
$$

Here $k s_{1}$ is the orientation angle between the helical fie ld and the vertical at the entrance, and $k s_{2}$ the angle at the end.

\section{Snakes and Rotators}

Snakes and/or rotators can be constructed by combining dipole and helical magnets. To design a snake or a rotator we have to satisfy the requirements that

(a) the orbit returns to the original values of $x, x^{\prime}, y, y^{\prime}$, i.e. the net deflection and displacement is zero;

(b) the spin transformation matrix, obtained by multiplying the appropriate matrices of forms (11) and (18) together, produces the desired spin rotation. Any spin transformation, i.e. any SU2 matrix, can be parametrized in the form

a

$$
\mathbf{M}=\exp \frac{i}{2} \mu(\vec{n} \cdot \vec{\sigma})
$$

where $\vec{n}$ is a unit vector.

For a snake (180 degree rotator) the parameter $\mu$ must equal $\pi$ or $180^{\circ}$, and the vector $\vec{n}$ (which is the axis of rotation) must lie in the horizontal plane; it is usually desirable for it to make an angle of $45^{\circ}$ with the longitudinal direction.

A sure way to ensure that the axis of rotation lie in the horizontal plane is to construct the snake or rotator with reflection symmetry, so that the vertical 
component of the field is antisymmetric about the center, while the horizontal field components are symmetric.

This is accomplished by adding to a set of magnets its reflection, consisting of the reflections of each of the elements in reverse order; the reflection matrix for each element is obtained by reversing the order of the factors and changing the sign of the coefficients of $\sigma_{3}$ but not of $\sigma_{1}$ and $\sigma_{2}$. Note that the reflection of a right-handed helix is also right-handed.

To make the spin at the crossing point longitudinal, as is necessary for experiments studying helicity dependence of interactions, one needs rotators that change the vertical spin in the arcsto horizontal at the crossing points, followed by the inverse rotator downstream from the crossing point. This may be accomplished by $a 90^{\circ}$ rotation about a horizontal axis (with the same symmetry as the snake described above), but other configurations are also possible. For example, the four-helix rotators described by $\mathrm{Ptitsin}^{3}$ do not have this reflection symmetry and do not have rotation angles of $90^{\circ}$, but they still rotate a vertical spin into the horizontal plane.

\section{Spreadsheet Calculations}

A Lotus-123 spreadsheet program has been written to evaluate the properties of snakes and rotators as functions of their parameters. A combination of horizontal and vertical (or tilted) deflector magnets and/or helix magnets is laid out, the spin matrices multiplied together, and the Lotus procedure "BSOLV" is applied to vary parameters so as to fit the constraints of zero orbit deflection and appropriate spin rotation.

This procedure has been applied to the four-helix snakes and rotators presently envisaged for the RHIC polarized proton project as described by Luccio ${ }^{4}$. We also investigate a "hybrid" configuraton, which consists of a single helix flanked by (horizontally deflecting) dipole magnets.

The procedure used here neglects nonlinear terms in the equations of motion, and also neglects fringing fields; therefore the numerical results for the four-helix configurations are not precisely identical with those given by Luccio, who solves differential equations both for orbital and for spin motion. But the simplified procedure lends itself to easy modification of parameters, and enables one to find parameters that optimize performance subject to given constraints. Once an optimal configuration has been found in this way, one may use the more exact differential equations for fine tuning.

The results, for the four-helix and for the hybrid configurations, are given in Table 1 and 2 and shown in Figures 1 through 4 . In each case we

${ }^{3}$ V. Ptitsin, RHIC/AP/49(Dec. 1994)

${ }^{4}$ A. Luccio, presentation to RHIC Polarized Proton Review, March 16, 1995 
show results both for injection energy $(\gamma=27)$ and maximum storage energy $(\gamma=268)$.

Note that the hybrid snake is significantly shorter than the helical snake, and that the helical magnet for the hybrid is longer than in the 4-helix case; this may make it easier to construct, and certainly reduces the effect of the nonlinear terms in the field (as can be seen from the fact that the nonlinear corrections to the magnetic fields $(1)$ are of the order $(k r)^{2}=(2 \pi r / \lambda)^{2}$, where $r$ is the orbit displacement and $\lambda$ is the helix twist wavelength, i.e. the length of a helical module. The maximum orbit excursion in the hybrid case is $10 \%$ more than in the helical case; this is probably not a serious drawback. It may therefore be advantageous to consider choosing the hybrid design rather than the 4-helix design for the snakes.

In the case of the rotators, on the other hand, the maximum excursion at injection ebergy is significantly larger than in the helical case; moreover the helical magnet has to be made with a short pitch, which may be difficult. The shorter length is hardly an advantage in this case, because the rotators are expected to be placed in the $Q 3-Q 4$ straight sections, which have plenty of room (they are $34 \mathrm{~m}$ long). Therefore the rotators should probably be made in the 4-helix mode as proposed in Luccio's report. 
Table 1: Four Helices

\begin{tabular}{|c|c|c|c|c|c|c|c|c|c|}
\hline Name & Description & $\gamma$ & $\begin{array}{l}B_{m x} \\
T\end{array}$ & $\begin{array}{l}\text { Lgth } \\
m\end{array}$ & $\begin{array}{l}\text { BL } \\
T-m\end{array}$ & $\begin{array}{l}\mathrm{xmx} \\
\mathrm{cm}\end{array}$ & $\begin{array}{l}\mathrm{ymx} \\
\mathrm{cm}\end{array}$ & $\begin{array}{l}\text { Axis } \\
\text { deg }\end{array}$ & $\begin{array}{l}\text { Rotn } \\
\text { deg }\end{array}$ \\
\hline \multirow[t]{2}{*}{$\begin{array}{l}\text { Helical } \\
\text { snake }\end{array}$} & $\begin{array}{l}4 \text { helices } \\
2.4 m \text { each }\end{array}$ & 27.0 & 3.96 & 10.56 & 24.9 & 1.37 & 2.96 & 45 & 180 \\
\hline & & 268 & 4.03 & 10.56 & 25.4 & 0.14 & 0.30 & 45 & 180 \\
\hline \multirow[t]{2}{*}{$\begin{array}{l}\text { Helical } \\
\text { rot }\end{array}$} & $\begin{array}{l}4 \text { helices } \\
2.4 m \text { each }\end{array}$ & 27.0 & 2.71 & 10.56 & 23.0 & 2.26 & 0.94 & 10.19 & 90 \\
\hline & & 268 & 3.52 & 10.56 & 32.5 & 0.38 & 0.12 & 101.2 & 90 \\
\hline
\end{tabular}

Table 2: Helix and 4 Dipoles

\begin{tabular}{llllllllll}
\hline Name & Description & $\gamma$ & $\begin{array}{l}B_{m x} \\
T\end{array}$ & $\begin{array}{l}\text { Lgth } \\
m\end{array}$ & $\begin{array}{l}\text { BL } \\
T-m\end{array}$ & $\begin{array}{l}\text { xmx } \\
\mathrm{cm}\end{array}$ & $\begin{array}{l}\text { ymx } \\
\mathrm{cm}\end{array}$ & $\begin{array}{l}\text { Axis } \\
\mathrm{deg}\end{array}$ & $\begin{array}{l}\text { Rotn } \\
\mathrm{deg}\end{array}$ \\
\hline $\begin{array}{l}\text { Hybrid } \\
\text { snake }\end{array}$ & $\begin{array}{l}\text { Full helix } \\
\text { \& 4 dipoles }\end{array}$ & 27.0 & 3.87 & 7.18 & 22.6 & 3.34 & 2.23 & 45 & 180 \\
& 268 & 3.95 & 7.18 & 23.0 & 0.34 & 0.23 & 45 & 180 \\
$\begin{array}{l}\text { Hybrid } \\
\text { rot }\end{array}$ & $\begin{array}{l}\text { Full helix } \\
\text { \& 4 dipoles }\end{array}$ & 27.0 & 3.84 & 7.38 & 23.4 & 3.95 & 1.01 & 10.19 & 90 \\
& & 268 & 3.91 & 7.38 & 23.8 & 0.13 & 0.10 & 101.2 & 90 \\
\hline
\end{tabular}


Four-helix snake, Energy $24.4 \mathrm{GeV}$

Helix fields 1.23 and 3.96 Tesla; axis 45.0 degrees from transverse

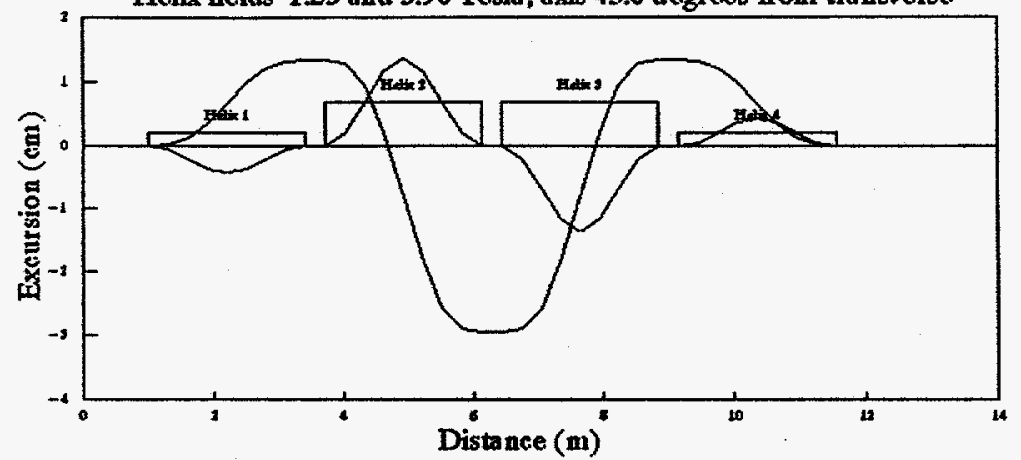

Figure 1

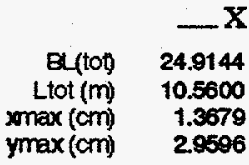

\begin{tabular}{|c|c|c|c|}
\hline Element & $\begin{array}{l}\text { Length B drection } \\
\text { (m) from horiz. }\end{array}$ & $\begin{array}{l}\text { B Fieid } \\
\text { (Testa) }\end{array}$ & $\underset{(T-m)}{B L}$ \\
\hline $\begin{array}{l}\text { Helix } \\
\text { Space }\end{array}$ & $\begin{array}{l}2.400-90 \text { to } 270 \\
0.320\end{array}$ & 1.232 & 2.557 \\
\hline $\begin{array}{l}\text { Hellx } \\
\text { Space } \\
\text { Hellx }\end{array}$ & $\begin{array}{l}2.400-2701090 \\
0.320 \\
2.400-90 \text { to } 270\end{array}$ & 3.958 & 9.500 \\
\hline $\begin{array}{l}\text { Space } \\
\text { Helix }\end{array}$ & $\begin{array}{l}0.320 \\
2.400-2701090\end{array}$ & 1.232 & 2.957 \\
\hline
\end{tabular}

Four-helix snake, Energy $250.5 \mathrm{GeV}$

Helix fields 1.26 and 4.03 Tesia; axis 45.0 degrees from transverse

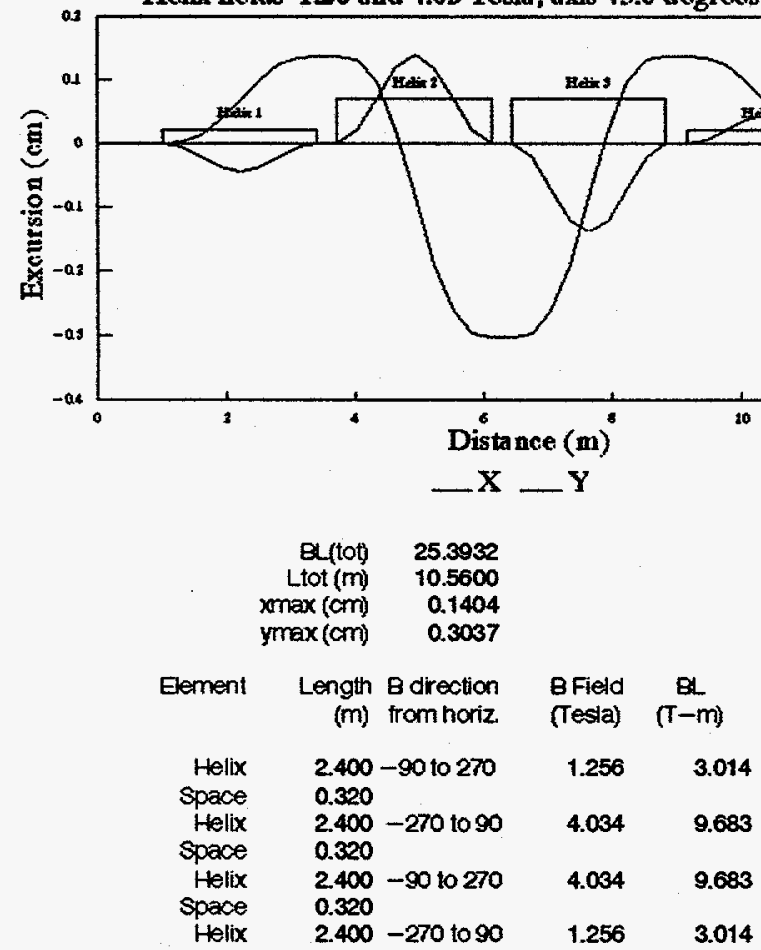


Four - helix rotator, ener gy $24.4 \mathrm{GeV}$

Helix fields 2.09 and 2.71 Tesla; spin 10.2 degrees from longitudinal

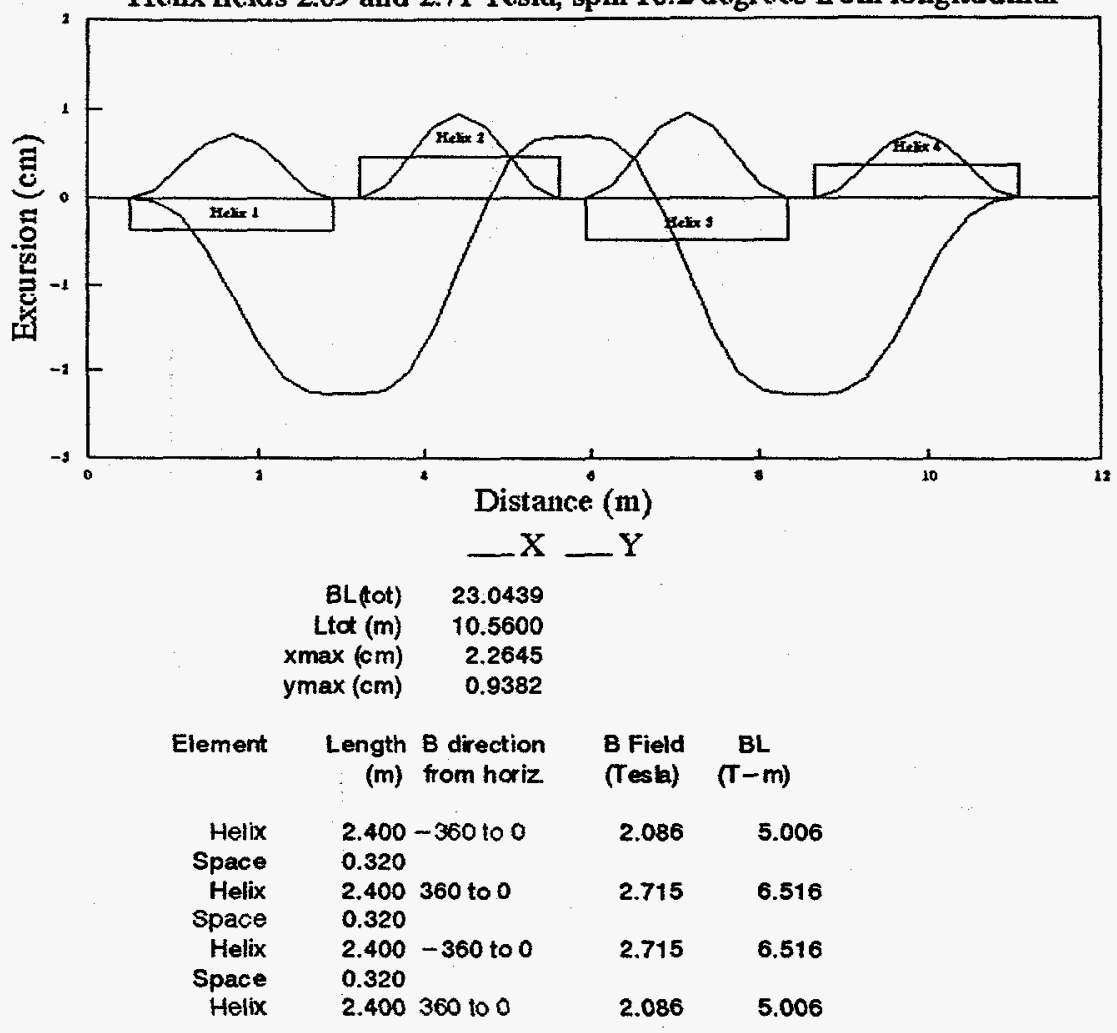

Figure 2

Four - helix rotator, energy $250.5 \mathrm{GeV}$

Helix fields 3.52 and 3.25 Tesla; spin 101.2 degrees from longitudinal

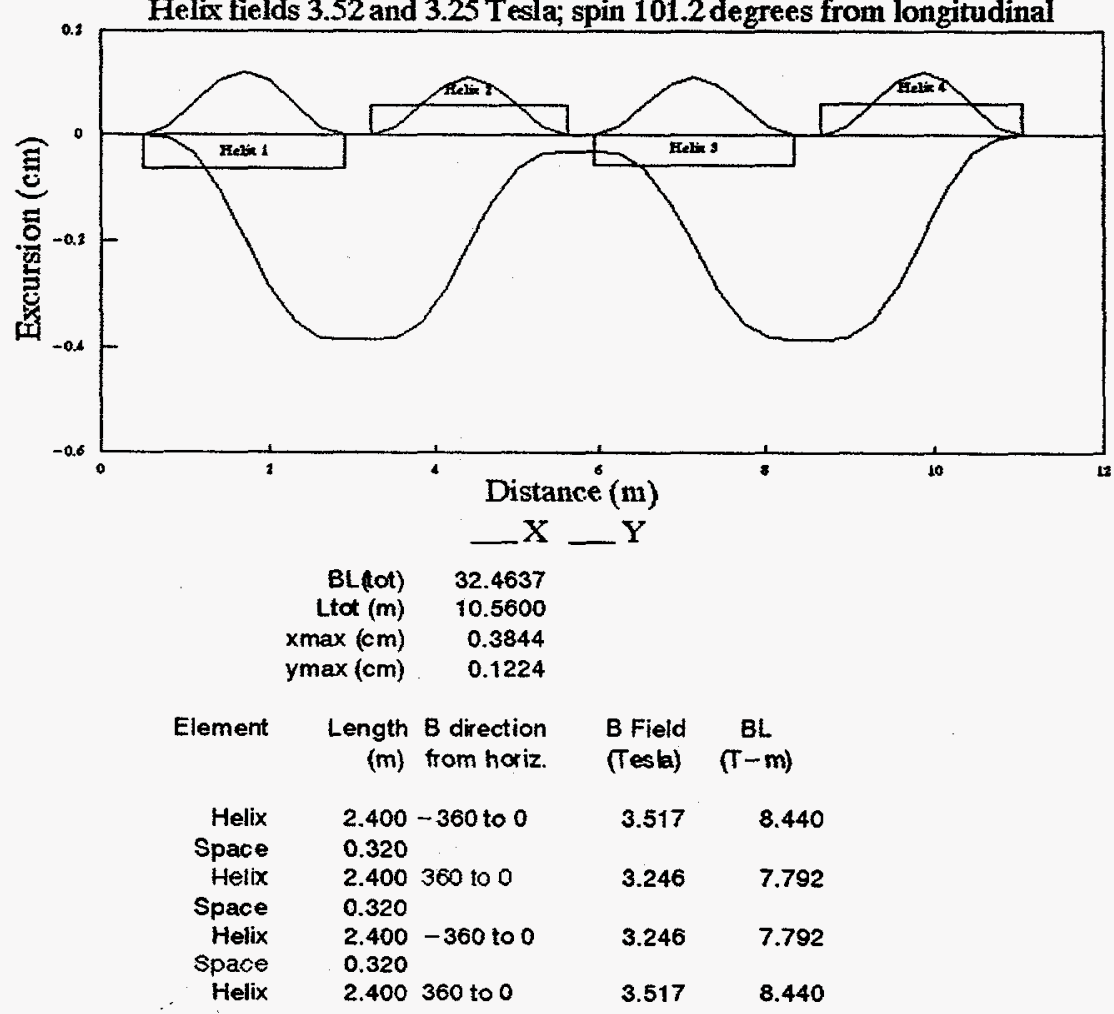


RHIC Snake, 3.87 T helix; 3.72 \& $3.85 T$ dipoles $24.4 \mathrm{GeV}$; axis 45.0 degrees

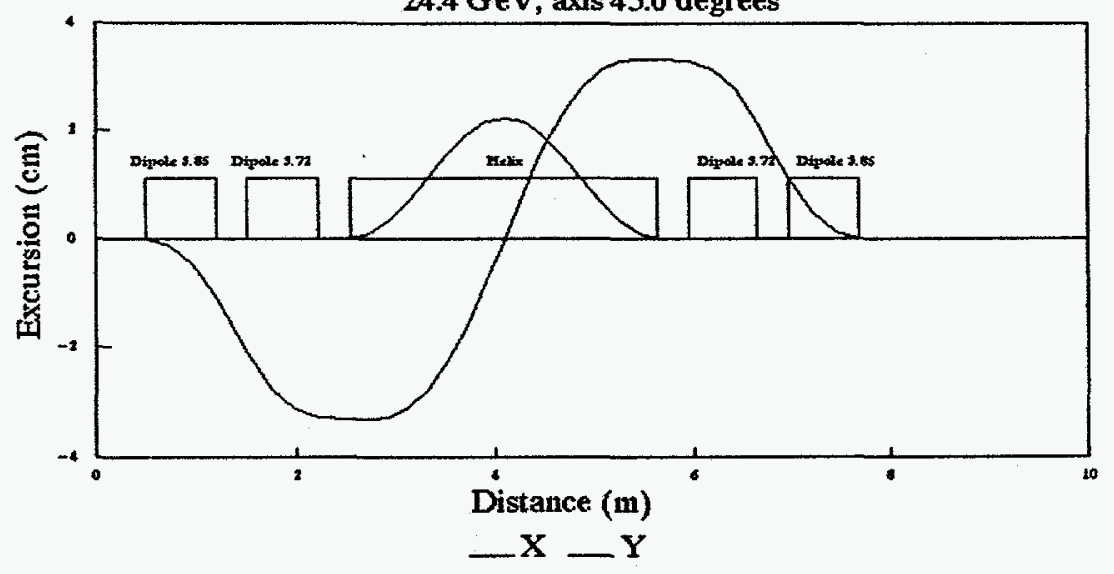

Figure 3

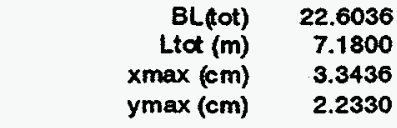

\begin{tabular}{|c|c|c|c|}
\hline Element & $\begin{array}{r}\text { Length } \\
\text { (m) }\end{array}$ & $\begin{array}{l}\text { B direction } \\
\text { from horiz. }\end{array}$ & $\begin{array}{l}\text { B Field } \\
\text { (Tesla) }\end{array}$ \\
\hline $\begin{array}{l}\text { Dipole } \\
\text { Space }\end{array}$ & $\begin{array}{l}0.700 \\
0.320\end{array}$ & 90.000 & 3.853 \\
\hline Dipole & 0.700 & -90.000 & 3.717 \\
\hline Space & 0.320 & & \\
\hline Helix & 3.100 & Oto 360 & 3.873 \\
\hline Space & 0.320 & & \\
\hline Dipole & 0.700 & 90.000 & 3.717 \\
\hline $\begin{array}{l}\text { Space } \\
\text { Dipole }\end{array}$ & $\begin{array}{l}0.320 \\
0.700\end{array}$ & -90.000 & 3.853 \\
\hline
\end{tabular}

RHIC Snake, 3.95 T helix; $3.79 \& 3.93 \mathrm{~T}$ dipoles $250.5 \mathrm{GeV}$; axis 45.0 degrees

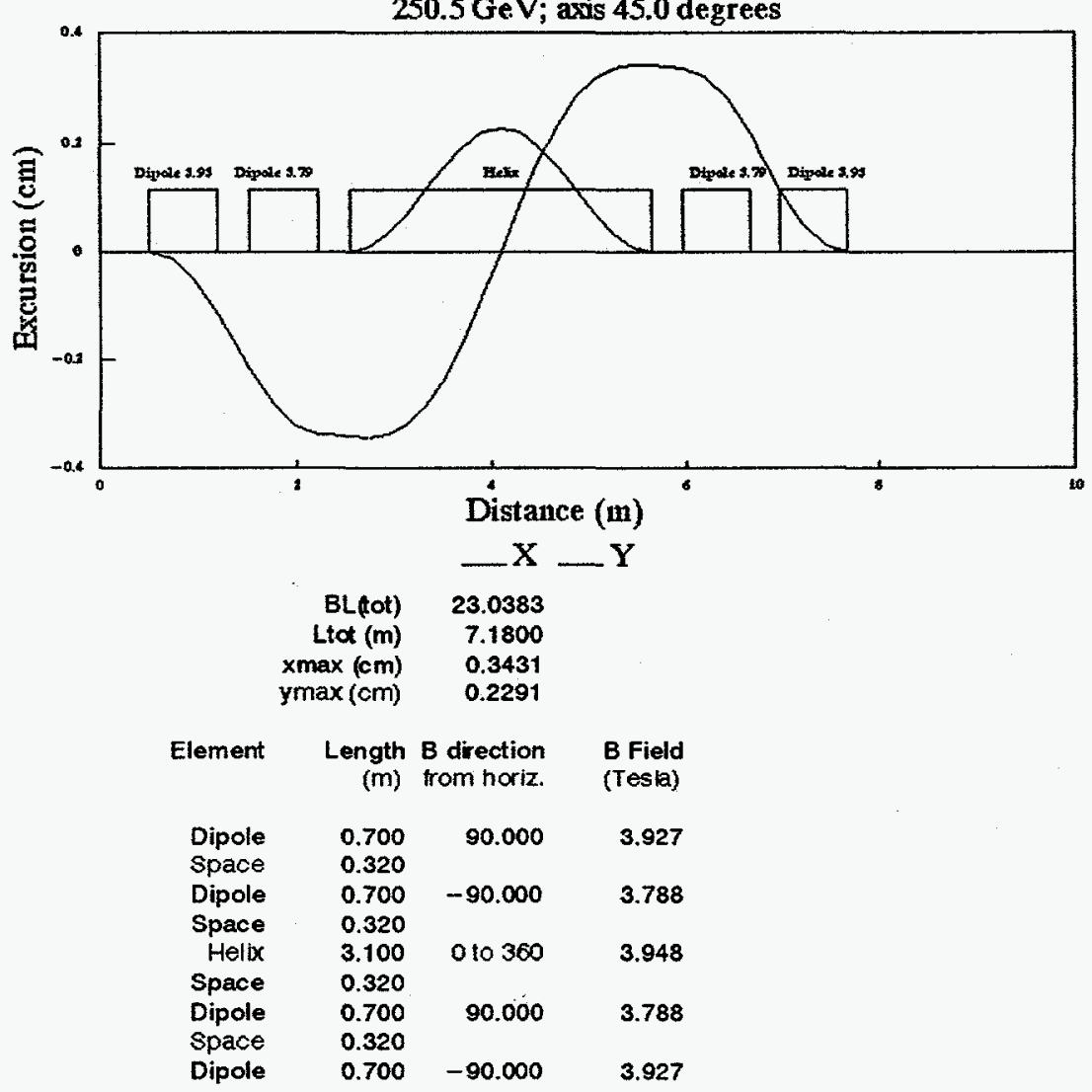


Rotator, 3.84 T Helix; 3.51 \& 3.66 T dipoles

$24.4 \mathrm{GeV}$; spin 10.2 degrees from longitudinal

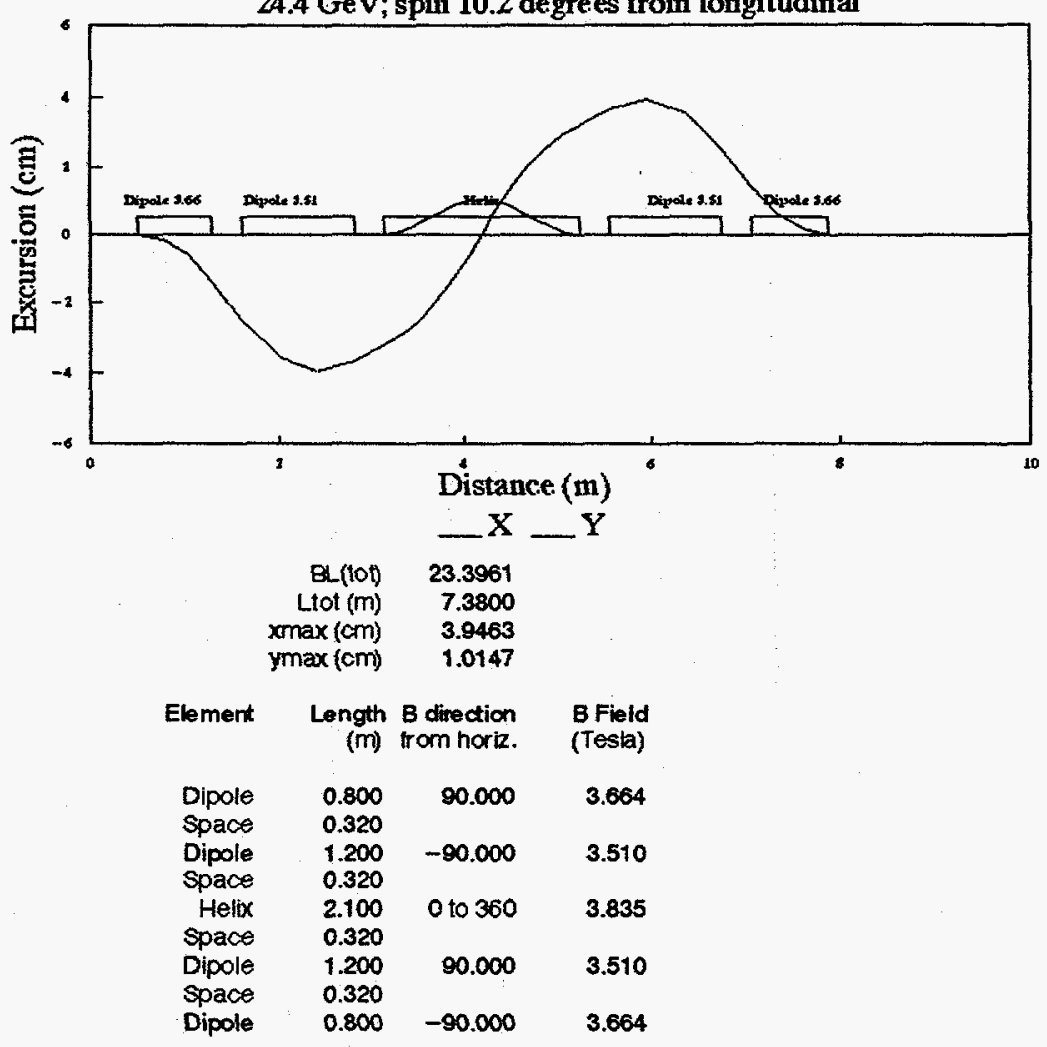

Figure 4

Rotator, 3.91 T Helix; $-2.17 \&-1.42$ T dipoles $250.5 \mathrm{GeV}$; spin 101.2 degrees from longitudinal

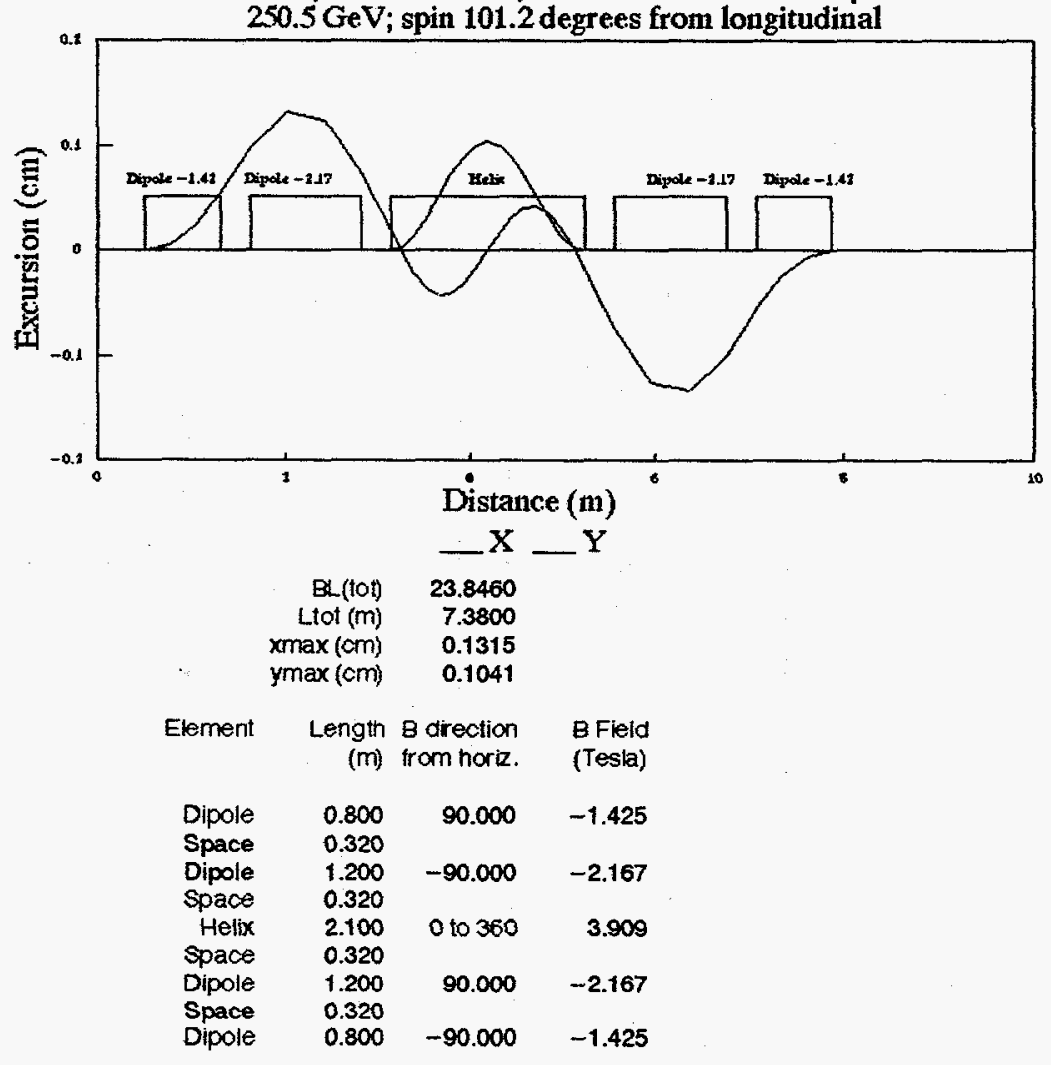

